\title{
Two step saccharification of xylan portion of sugarcane waste by recombinant xylanolytic enzymes for enhanced xylose production
}

Abhijeet Thakur ${ }^{1, \dagger}$, Aakash Sharma ${ }^{1, \dagger}$, Kaustubh Chandrakant Khaire ${ }^{2}$, Vijayanand Suryakant Moholkar $^{2,3}$, Puneet Pathak ${ }^{4}$, Nishi Kant Bhardwaj ${ }^{4}$ and Arun Goyal ${ }^{1,2, *}$

${ }^{1}$ Carbohydrate Enzyme Biotechnology Laboratory, Department of Biosciences and Bioengineering, ${ }^{2}$ Center for energy, ${ }^{3}$ Department of Chemical Engineering, Indian Institute of Technology Guwahati, Guwahati-781039, India. ${ }^{4}$ Avantha Centre for Industrial Research \& Development, Paper Mill Campus, Yamuna Nagar - 135001, Haryana, India.

\section{$\dagger$ Equal contribution}

\section{*Corresponding author}

Professor Arun Goyal

Department of Biosciences and Bioengineering, Indian Institute of Technology Guwahati,

Guwahati-781039, India

Tel: +91-361-258-2208

E mail: arungoyl@iitg.ac.in 
Figure S1 3-D response surface plots for the interaction between the independent variables involved in the optimization of pretreatment. A) Pretreatment optimization for SB, B) pretreatment optimization for SCT. (i)Temperature and ammonia conc., (ii) Time and ammonia conc., (iii) $\mathrm{S} / \mathrm{L}$ ratio and ammonia conc., (iv) Time and temperature, (v) S/L ratio and temperature and (vi) $\mathrm{S} / \mathrm{L}$ ratio and time.

A
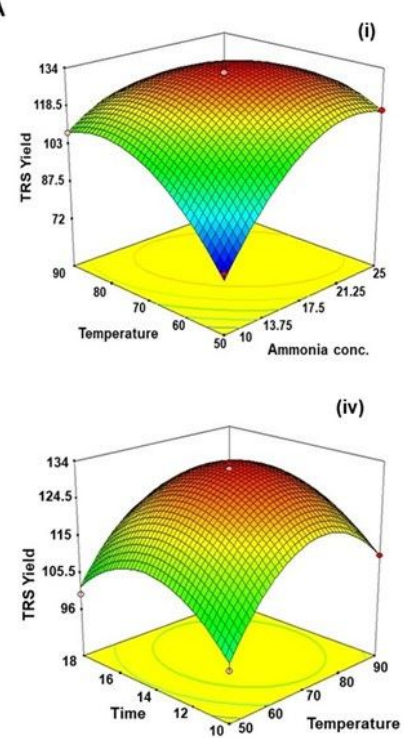

B
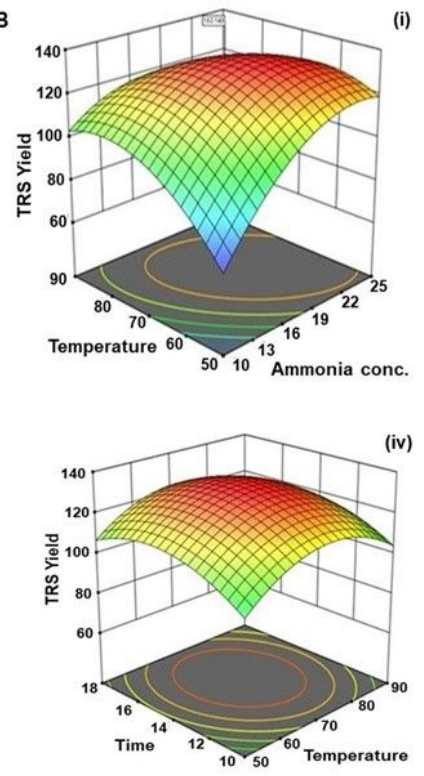
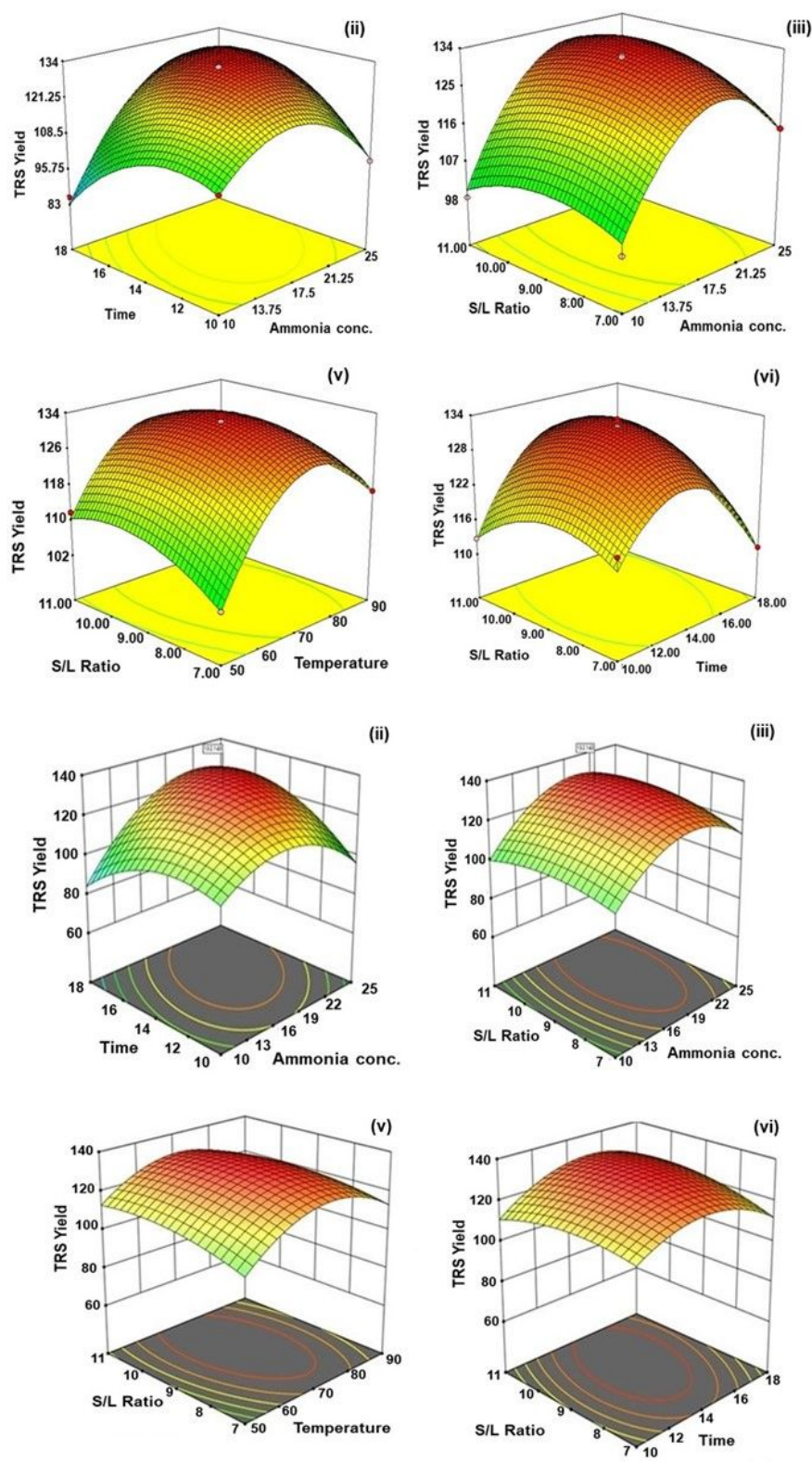
Table S1. Box-Behnken design and response for hemicellulose hydrolysis from pretreated SB by endo-1,4- $\beta$-xylanase (CtXyn11A) and $\alpha$-L-arabinofuranosidase (PsGH43_12).

\begin{tabular}{ccccc}
\hline $\begin{array}{c}\text { Run } \\
\text { order }\end{array}$ & $\begin{array}{c}\text { Biomass } \\
\text { loading } \\
(\%, \mathrm{w} / \mathrm{v})\end{array}$ & $\begin{array}{c}\text { Xylanase loading } \\
(\mathrm{U} / \mathrm{g} \text { ptd biomass }) \\
\left(\mathrm{A}_{1}\right)\end{array}$ & $\begin{array}{c}\text { Arabinofuranosidase } \\
\text { loading } \\
\text { (U/g })\end{array}$ & $\begin{array}{c}\mathrm{TRS}_{(\mathrm{XOS})} \text { yield } \\
(\mathrm{mg} / \mathrm{g} \text { ptd biomass })\end{array}$ \\
\hline 1 & 3 & 275 & 110 & 89.74 \\
2 & 5 & 275 & 20 & 72.2 \\
3 & 5 & 500 & 110 & 76.5 \\
4 & 1 & 275 & 20 & 79.1 \\
5 & 3 & 275 & 110 & 90.08 \\
6 & 3 & 500 & 20 & 90.9 \\
7 & 3 & 275 & 110 & 88.03 \\
8 & 1 & 500 & 110 & 90.6 \\
9 & 3 & 275 & 110 & 90.4 \\
10 & 3 & 500 & 200 & 91.9 \\
11 & 3 & 275 & 110 & 90.9 \\
12 & 3 & 50 & 20 & 62.2 \\
13 & 5 & 275 & 200 & 71.1 \\
14 & 1 & 50 & 110 & 65.3 \\
15 & 1 & 275 & 200 & 85.9 \\
16 & 3 & 50 & 200 & 75.9 \\
17 & 5 & 50 & 110 & 57.6 \\
\hline
\end{tabular}


Table S2. Box-Behnken design and response for hemicellulose hydrolysis from pretreated SCT by endo-1,4- $\beta$-xylanase (CtXyn11A) and $\alpha$-L-arabinofuranosidase (PsGH43_12).

\begin{tabular}{|c|c|c|c|c|}
\hline $\begin{array}{l}\text { Run } \\
\text { order }\end{array}$ & $\begin{array}{c}\text { Biomass loading } \\
(\%, \mathrm{w} / \mathrm{v}) \\
\left(\mathrm{A}_{2}\right)\end{array}$ & $\begin{array}{c}\text { Xylanase loading } \\
\text { (U/g ptd biomass) } \\
\left(\mathrm{B}_{2}\right)\end{array}$ & $\begin{array}{l}\text { Arabinofuranosidase } \\
\text { loading } \\
\text { (U/g ptd biomass) }\left(\mathrm{C}_{2}\right)\end{array}$ & $\begin{array}{c}\mathrm{TRS}_{(\mathrm{XOS})} \text { yield } \\
\text { (mg/g ptd } \\
\text { biomass) }\end{array}$ \\
\hline 1 & 3 & 275 & 110 & 82.04 \\
\hline 2 & 5 & 275 & 20 & 66.2 \\
\hline 3 & 5 & 500 & 110 & 69.6 \\
\hline 4 & 1 & 275 & 20 & 71.2 \\
\hline 5 & 3 & 275 & 110 & 81.64 \\
\hline 6 & 3 & 500 & 20 & 82.3 \\
\hline 7 & 3 & 275 & 110 & 80.78 \\
\hline 8 & 1 & 500 & 110 & 83.03 \\
\hline 9 & 3 & 275 & 110 & 79.8 \\
\hline 10 & 3 & 500 & 200 & 85.6 \\
\hline 11 & 3 & 275 & 110 & 81.9 \\
\hline 12 & 3 & 50 & 20 & 59.5 \\
\hline 13 & 5 & 275 & 200 & 64.7 \\
\hline 14 & 1 & 50 & 110 & 60.46 \\
\hline 15 & 1 & 275 & 200 & 78.5 \\
\hline 16 & 3 & 50 & 200 & 70.5 \\
\hline 17 & 5 & 50 & 110 & 55.46 \\
\hline
\end{tabular}


Table S3. ANOVA for quadratic model of hemicellulose hydrolysis from pretreated SB by endo-1,4- $\beta$-xylanase (CtXyn11A) and $\alpha$-L-arabinofuranosidase (PsGH43_12).

\begin{tabular}{lllllll}
\hline Source & SS & Df & Mean square & F-Value & p-value & \\
\hline Quadratic model & 2081.09 & 9 & 231.23 & 108.36 & $<0.0001$ & Significant \\
A $_{1}$-Biomass loading & 236.53 & 1 & 236.53 & 110.84 & $<0.0001$ & \\
$\mathrm{~B}_{1}$-Xylanase loading & 987.90 & 1 & 987.90 & 462.94 & $<0.0001$ & \\
C $_{1}$-Arabinofuranosidase & & & & & & \\
loading & 52.02 & 1 & 52.02 & 24.38 & 0.0017 & \\
$\mathrm{~A}_{1} \mathrm{~B}_{1}$ & 10.24 & 1 & 10.24 & 4.80 & 0.0646 & \\
$\mathrm{~A}_{1} \mathrm{C}_{1}$ & 15.60 & 1 & 15.60 & 7.31 & 0.0305 \\
$\mathrm{~B}_{1} \mathrm{C}_{1}$ & 40.32 & 1 & 40.32 & 18.90 & 0.0034 \\
$\mathrm{~A}_{1}{ }^{2}$ & 441.51 & 1 & 441.51 & 206.89 & $<0.0001$ & \\
$\mathrm{~B}_{1}{ }^{2}$ & 211.66 & 1 & 211.66 & 99.18 & $<0.0001$ & \\
$\mathrm{C}_{1}{ }^{2}$ & 14.76 & 1 & 14.76 & 8.11 & 0.0096 \\
Residual & 14.94 & 7 & 2.13 & & \\
Lack of fit & 10.16 & 3 & 3.39 & 2.83 & 0.1701 \\
Pure error & 4.78 & 4 & 1.20 & & \\
Model statistics & & & & & \\
S.D. & 1.46 & & $\mathrm{R}^{2}$ & & \\
Mean & 80.49 & & Adj-R & & \\
C.V. \% & 1.81 & & Pred-R & 0.9929 & & \\
\hline
\end{tabular}


Table S4. ANOVA for quadratic model of hemicellulose hydrolysis from pretreated SCT by endo-1,4- $\beta$-xylanase (CtXyn11A) and $\alpha$-L-arabinofuranosidase (PsGH43_12).

\begin{tabular}{|c|c|c|c|c|c|c|}
\hline Source & SS & Df & Mean square & F-Value & $\mathrm{p}$-value & \\
\hline Quadratic model & 1480.27 & 9 & 164.47 & 90.33 & $<0.0001$ & Significant \\
\hline $\mathrm{A}_{2}$-Biomass loading & 173.26 & 1 & 173.26 & 95.15 & $<0.0001$ & \\
\hline $\mathrm{B}_{2}$-Xylanase loading & 695.83 & 1 & & 382.15 & $<0.0001$ & \\
\hline $\mathrm{C}_{2}$-Arabinofuranosidase & & & & & & \\
\hline loading & 50.50 & 1 & 50.50 & 27.14 & 0.0012 & \\
\hline $\mathrm{A}_{2} \mathrm{~B}_{2}$ & 17.77 & 1 & 17.77 & 9.76 & 0.0168 & \\
\hline $\mathrm{A}_{2} \mathrm{C}_{2}$ & 19.36 & 1 & 19.36 & 10.63 & 0.0139 & \\
\hline $\mathrm{B}_{2} \mathrm{C}_{2}$ & 14.82 & 1 & 14.82 & 8.14 & 0.0246 & \\
\hline $\mathrm{A}_{2}^{2}$ & 357.13 & 1 & 357.13 & 196.14 & $<0.0001$ & \\
\hline $\mathrm{B}_{2}^{2}$ & 100.47 & 1 & 100.47 & 55.18 & 0.0001 & \\
\hline $\mathrm{C}_{2}^{2}$ & 14.76 & 1 & 14.76 & 8.11 & $<0.0248$ & \\
\hline Residual & 12.75 & 7 & 1.82 & & & \\
\hline Lack of fit & 9.23 & 3 & 3.08 & 3.49 & 0.1291 & \\
\hline Pure error & 1.02 & 4 & 0.25 & & & \\
\hline \multicolumn{7}{|l|}{ Model statistics } \\
\hline S.D. & 1.35 & & $\mathrm{R}^{2}$ & 0.9915 & & \\
\hline Mean & 73.72 & & $\operatorname{Adj}-R^{2}$ & 0.9805 & & \\
\hline C.V. \% & 1.83 & & Pred-R ${ }^{2}$ & 0.8975 & & \\
\hline
\end{tabular}


Table S5. Hemicellulose content and TRS yield after each step.

\begin{tabular}{|c|c|c|c|c|}
\hline Steps followed & SB & $\begin{array}{c}\% \text { of SB } \\
\text { conversion }\end{array}$ & SCT & $\begin{array}{l}\% \text { of SCT } \\
\text { conversion }\end{array}$ \\
\hline $\begin{array}{l}\text { Hemicellulose content in } \\
\text { raw biomass }\end{array}$ & $28.6 \mathrm{~g} / 100 \mathrm{~g}$ & - & $27.4 \mathrm{~g} / 100 \mathrm{~g}$ & - \\
\hline $\begin{array}{l}\text { Hemicellulose content in } \\
\text { optimized SAA pretreated } \\
\text { biomass }\end{array}$ & $17.5 \mathrm{~g} / 78 \mathrm{~g}$ & - & $15.5 \mathrm{~g} / 75 \mathrm{~g}$ & - \\
\hline $\begin{array}{l}\text { TRS }_{(\mathrm{XOS})} \text { yield after first step } \\
\text { saccharification }\end{array}$ & $\begin{array}{l}93.2 \mathrm{mg} / \mathrm{g} \mathrm{ptd} \mathrm{SB} \\
(3.2 \mathrm{mg} / \mathrm{ml})\end{array}$ & - & $\begin{array}{l}85.1 \mathrm{mg} / \mathrm{g} \mathrm{ptd} \mathrm{SCT} \\
(2.5 \mathrm{mg} / \mathrm{ml})\end{array}$ & - \\
\hline $\begin{array}{l}\text { TRS yield after second step } \\
\text { saccharification }\end{array}$ & $\begin{array}{c}164.7 \mathrm{mg} / \mathrm{g} \text { ptd SB } \\
(12.9 \mathrm{~g} \mathrm{TRS} / 17.5 \mathrm{~g} \\
\text { of hemicellulose) }\end{array}$ & 73.5 & $\begin{array}{c}147.2 \mathrm{mg} / \mathrm{g} \text { ptd SCT } \\
(11.03 \mathrm{~g} \mathrm{TRS} / 15.5 \mathrm{~g} \\
\text { of hemicellulose })\end{array}$ & 71.1 \\
\hline $\begin{array}{l}\text { TFA hydrolysis of ptd biomass } \\
\text { (total xylose by HPLC) }\end{array}$ & $173 \mathrm{mg} / \mathrm{g}$ ptd SB & - & $139 \mathrm{mg} / \mathrm{g}$ ptd SCT & - \\
\hline $\begin{array}{l}\text { TFA hydrolysis of ptd biomass } \\
\text { (total arabinose by HPLC) }\end{array}$ & $21 \mathrm{mg} / \mathrm{g}$ ptd SB & & $27 \mathrm{mg} / \mathrm{g}$ ptd SCT & \\
\hline $\begin{array}{l}\text { Second step enzymatic } \\
\text { hydrolysis (xylose by HPLC) }\end{array}$ & $120.4 \mathrm{mg} / \mathrm{g}$ ptd SB & - & $89.2 \mathrm{mg} / \mathrm{g}$ ptd SCT & - \\
\hline $\begin{array}{l}\text { Second step enzymatic } \\
\text { hydrolysis (arabinose by } \\
\text { HPLC) }\end{array}$ & $14 \mathrm{mg} / \mathrm{g}$ ptd SB & - & $18.9 \mathrm{mg} / \mathrm{g}$ ptd $\mathrm{SCT}$ & - \\
\hline Xylan to xylose conversion & $120.4 \mathrm{mg} / 173 \mathrm{mg}$ & 69.6 & $89.2 \mathrm{mg} / 139 \mathrm{mg}$ & 64.1 \\
\hline
\end{tabular}

